\title{
Enabling Smart Navigation by Incorporating Human Inspired Evaluation of Route Sections
}

\author{
Christian Brandstaetter ${ }^{1,2 *}$, Martin Fittner ${ }^{1}$ \\ 1Institute of Computer Technology at Vienna University of Technology, Gußhausstraße 27-29, 1040 Vienna. \\ ${ }^{2}$ Research Institute of Molecular Pathology (IMP), Dr. Bohr-Gasse 7, 1030 Vienna. \\ * Corresponding author. Email: brandstaetter@imp.ac.at \\ Manuscript submitted January 18, 2017; accepted April 9, 2017. \\ doi: 10.17706/jcp.13.2.187-193
}

\begin{abstract}
It is challenging for every child not becoming lost in the shopping center. Simulated agents have the same problem. So they need some localization. On the basis of the existing cognitive architecture in th project Simulation of Mental Apparatus and Applications (SiMA) at the Institute of Computer Technology (ICT) currently, we show, how cognitive architectures can get extended by location awareness and navigation in a human-like way. This paper deals with particular requirements for the used data structures to enable self-localization and pathfinding in a bionically inspired way. The typical data structure for navigation in the form of a connected graph gets modified and extended to be compatible with psychoanalytic concepts and still allow navigation. The functionality was implemented exemplary in a multiagent simulation.
\end{abstract}

Key words: Artificial general intelligence, artificial recognition system, cognitive architectures, cognitive automation, landmarks, localization, navigation, psychoanalytically inspired ai, software agents.

\section{Introduction}

The project SiMA was founded by Dietmar Dietrich [1] by introducing a bionic approach to the field of building automation because of the rising demands there. The science of bionics describes the way of finding new ideas for useful artificial machines and systems by examining the nature of living systems to take inspiration from [2]. The projects verification tool, an artificial life simulation in which the software agents are placed into, showed us an amazing circumstance. Agents act kind of draft when not knowing spatial relations of objects in their surrounding environment. They pass by a valuable food source, without being hungry. A short time later, they get hungry, but do not remember where to find the food source. When discovering an enemy, from which they want to flee for whatever reason, they turn around and run away. But a short time after losing the enemy from their field of vision, they lose their fear and decide to move around and seek for somewhat. Accidental they move into the direction of the enemy and the loop starts for a new round. Hence, a clear demand exists for a method to put the perception and experiences into locational relation to each other.

\section{State of the Art}

Existing Algorithms for Mapping, Self-Localization, and Path Finding have already been discussed in [3]. In the current paper at hand, we would like to outline what is necessary in addition to the right algorithms. This is a data structure for navigation enabling processing in a human-like way. Let us give a short introduction to 
psychoanalysis, what all of that is based upon.

\subsection{Psychoanalysis}

Psychoanalysis is a procedure for investigating mental processes using free association, dream interpretation, and interpretation of resistance and transference manifestations. It was devised by Sigmund Freud in the 1890s. Psychoanalysis consists of psychological and psychotherapeutic techniques and theories. After Erich Fromm [4] the basic pillars of Psychoanalysis can be summarized as follows.

- A person's development is determined by often forgotten events in early childhood rather than by inherited traits alone.

- Human attitude, mannerism, experience, and thought is largely influenced by irrational drives that are rooted in the unconscious.

- It is necessary to bypass psychological resistance in the form of defense mechanisms when bringing drives into awareness.

- Conflicts between the conscious and the unconscious, or with the repressed material can materialize in the form of mental or emotional disturbances, for example neurosis, neurotic traits, anxiety, depression, etc.

- Liberating the elements of the unconscious is achieved through bringing this material into the conscious mind (via e.g. skilled guidance, i.e. therapeutic intervention).

\subsection{Schools and Theories of Psychoanalysis}

Over time a lot of several theoretical schools have been developed by forking from the initial ideas after Sigmund Freud. Even as they differ in wide areas, most of them state, that there is an influence of unconscious elements on the conscious. Attempts have been made to consolidate elements of conflicting theories of psychoanalysis to make them comparable [5].

Topographic theory was defined by Sigmund Freud in The Interpretation of Dreams [6]. The quintessence of the Topographic theory is that the mental apparatus can be divided into Conscious, Preconscious, and Unconscious. These are mental processes and not anatomical structures of the brain. Basically, Sigmund Freud retained this theory throughout his life but he later replaced it in large areas by the Structural theory [7]. When someone today wants to describe how the mind functions in classical psychoanalytic theory he most likely uses the Topographic theory as one of the meta-psychological points of view.

Structural theory was defined by Sigmund Freud in The Ego and the Id [8]. It divides the psyche into id, ego, and superego. An illustration of id, ego, and superego can be found in Fig. 1.

\subsection{Implementation of Psychoanalysis}

- From the human decision-making process, the functional components have been extracted and specified in detail by functions and the definition of their in-between interfaces in a top-down analysis and have been implemented in an autonomous agent's decision-making process. The result is a psychoanalytically inspired architecture. It consists of a primary and a secondary process. In the secondary process thoughts are conscious while the primary process is completely unconscious. The psychoanalytical theories of the current SiMAi16-Framework [9] are tested by using an autonomous agent simulation. The result is a modular framework with an integrated evaluation system which considers internal and external assessment criteria. Especially when it comes to data interpretation and evaluation using internal and external performance indicators, the implementation of the agent in the simulated environment shows the strengths of the system. A more detailed description can be found in [10]. The framework implements for each simulated agent a rash of modules, modeling the human brain in a psychoanalytic way. Splitting of the cognitive process of the mind into the primary and the secondary process is based on the method, how the 
information is represented and handled.

- The primary process only takes internal drive demands into account and is, therefore, independent of the current context and the situation the agent is in. Processing of information happens regardless of possible conflicts and contradictions contained within it. Sigmund Freud defined the pleasure principle after that the primary process is working. It means being driven by the aim to satisfy basics needs no matter what. Things presentation and affects are manipulated by the primary process. There are no logical relations defined on them, and no reasoning is done.

- The secondary process processes the information and assigns logical, local and temporal associations. Conflicts and contradictions that are found within the information during the processing are resolved. The current situation and rule are taken into consideration. Sigmund Freud defined the reality principle after that the secondary process is working. Word presentations are manipulated by the secondary process. There are logical, local and temporal associations allowing high-level reasoning.

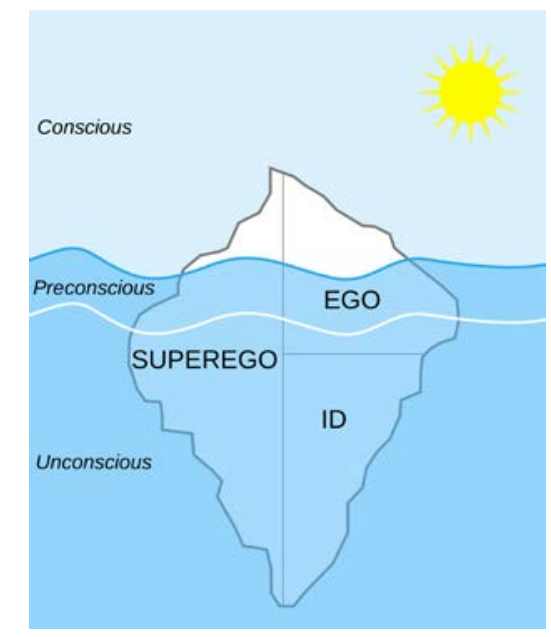

Fig. 1. ID, ego and superego in the structural theory of sigmund freud.

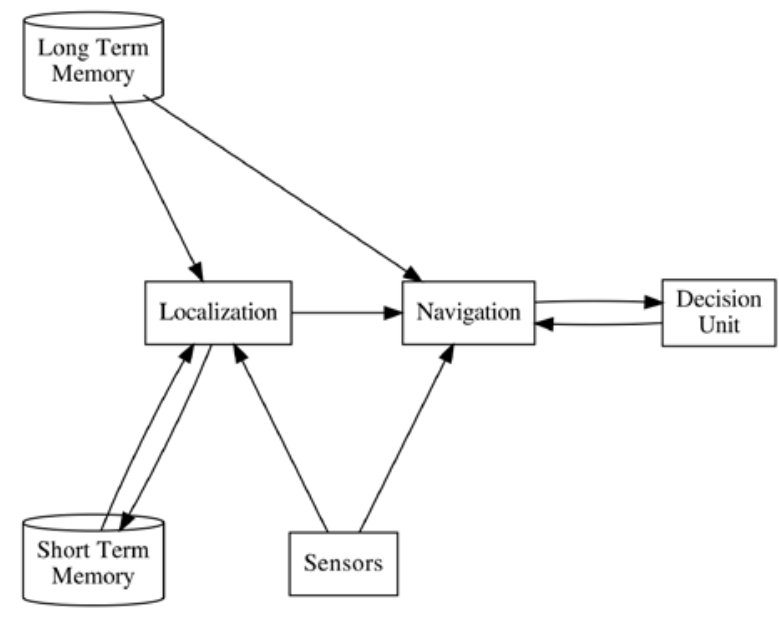

Fig. 2. Process of localization and navigation in SiMA.

The work at hand is part of the secondary process of the SiMA model which is the environment in which localization and navigation routines take place.

\section{Navigation in the SiMA Project}

Technically the Problem of Simultaneous Localization and Mapping (SLAM) is already solved. Maybe there is room for optimizations, but that is not the scope of this work. In this work, we introduce the concept of SLAM to the existing model of the project SiMA which has the particular limitation to operate after the archetype of the human psyche and is inspired by the second topographical model of Sigmund Freud.

A rough overview of the involved components is depicted in Fig. 2.

The Model consists of:

- Two databases (Long- and Short-Term Memory)

- Two internal Processes (Localization and Navigation)

- Two external Units (Decision Unit and Sensors)

\section{Psychoanalytically Inspired Data Structures for Navigation}

Any intelligent agent needs to reasonable act in an environment, an internal representation of its surroundings. The concept of spatial knowledge representation comes from the field of cognitive science, 
first described in an article by Tolman 1948 [11] designated as a cognitive map, and has since that time been the subject of extensive research. It has not yet reached a general consensus regarding the definition. Nevertheless, this concept has developed over the years and became an important part of many other areas of science, such as geography, urban planning, and psychology.

\subsection{Requirements}

In the human brain, no exact numbers with kilometer or meter exist. The human brain has a relation-mesh and sets new distances in relation to its known distances. This relation is grounded in the body of the human being. So relations like a glass is a little bit bigger or smaller than my hand, and a one family house is in general 3-5 times bigger than I am. With these relations, we build up millions of relations that of course are grounded to the own body. So without a body or better without the knowledge of having a body, we would never get the idea to build up distances and relate them together.

For mapping different locations and to get a feeling how far they are we search in our memories for the most similar available location relation we have learned in our life and take over the learned feeling. When the decision then doesn't lead to the expected results we adapt that differences in relation to other changes we made in situations of deviations in our past.

\subsection{What to Modify at Existing Data Models?}

Classic navigation systems use data in the form of a graph, consisting of edges and nodes. The distance between the nodes is encoded as the weight of the edge. A conceivable database can be seen in Fig. 3 .

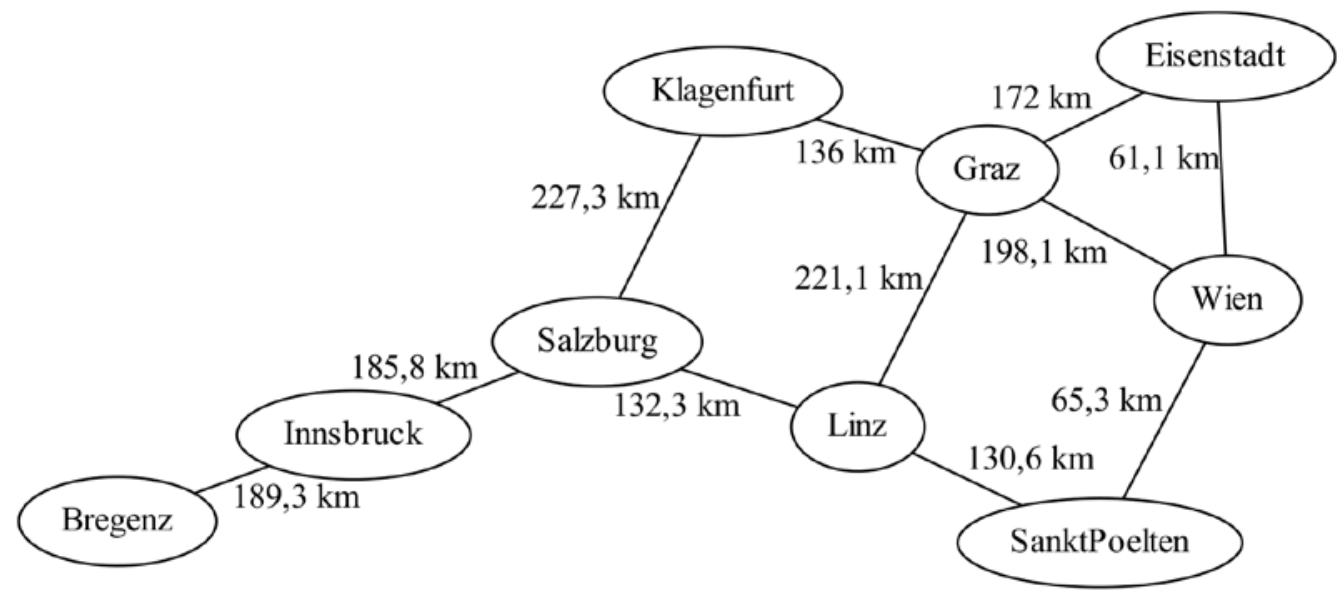

Fig. 3. Database of classic navigation systems.

Some navigation systems do not only include the distance of the segments but also the estimated time of traveling of the segment. New ways of offboard navigation like Google maps also include traffic information for including traffic jams in their calculation. However, what all of them lack is the preference of the driver. The major advance, what We like to propose in the work at hand, is to include driver preferences in the navigation process. On one hand side the general attitude of the driver, which will be somehow long term persistent. Moreover, on the other hand side the mood of the driver, which is more short term. For enabling this it is not possible to use the static distance of the segment of the way as the weight of the edge anymore. It is necessary to calculate the weight of the edges based on a number of values. The general attitude of the driver comes from the character parameters and the information about the mood of the driver comes from the emotions in the primary process [12].

Depending on our experiences in the past, we condensed them to feelings. Feelings in the SiMA project 
should be understood as high-level evaluation of valuations of past experiences. So feelings form a decision proposal for related situations without any further time-consuming calculation of situation dependent circumstances. For example, if we travel with a tram and we take a seat and realize later that dirt on the seat messed our clothes we will have a very bad feeling about that tram, and we would avoid going by tram in the future. However, there will be quite more feelings like an overall degree of the positive attitude to public transport and a bad feeling about trams which is not as bad as about the specific tram line where that bad situation has happened.

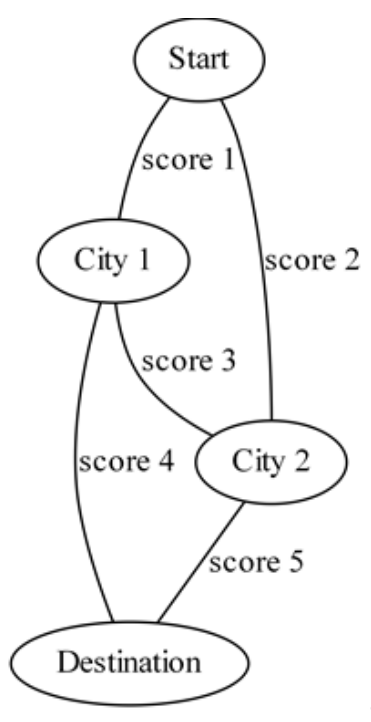

Fig. 4a. No route selected.

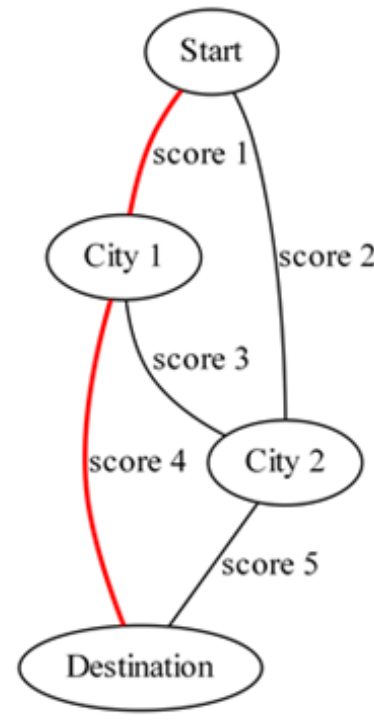

Fig. 4b. Route 1 selected.

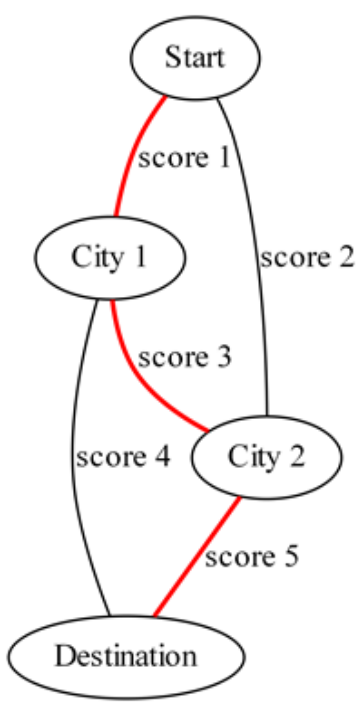

Fig. 4c. Route 2 selected.

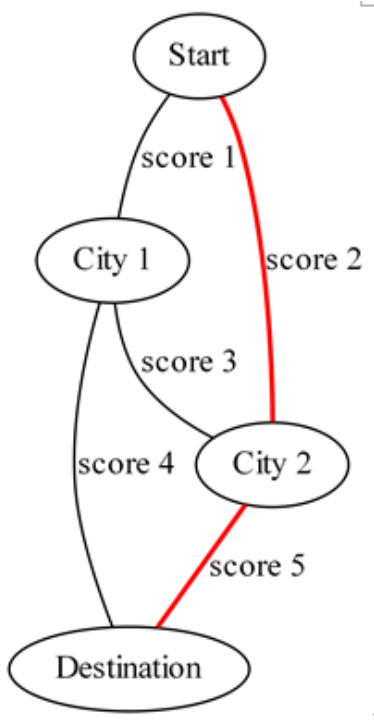

Fig. 4 d. Route 3 selected.

Fig. 4. Brain-like data model for navigation.

Table 1. Examples of the Different Scores

\begin{tabular}{|c|c|c|c|}
\hline & Street-Type & Experienced situations & Formula \\
\hline Score 1 & Normal street & no special experience & $\begin{array}{l}\text { Just based on distance or traveling } \\
\text { time }\end{array}$ \\
\hline Score 2 & Motorway & cool for driving fast & $\begin{array}{l}\text { distance or traveling time plus a } \\
\text { bonus when the agent is aggressive }\end{array}$ \\
\hline Score 3 & $\begin{array}{l}\text { Street with lots of } \\
\text { serpentines and } \\
\text { bridges with overlooks }\end{array}$ & Nice for a relaxing car ride & $\begin{array}{l}\text { distance or traveling time plus a } \\
\text { bonus when the agent is in a } \\
\text { relaxing mood }\end{array}$ \\
\hline Score 4 & $\begin{array}{l}\text { Long tunnel with } \\
\text { two-way traffic }\end{array}$ & $\begin{array}{l}\text { Fast, because of direct connection, } \\
\text { but exhausting when being tired }\end{array}$ & $\begin{array}{l}\text { distance or traveling time plus a } \\
\text { bonus when the agent is in a hurry } \\
\text { and not tired }\end{array}$ \\
\hline Score 5 & Car slepper train & $\begin{array}{l}\text { Super relaxing, can sleep while } \\
\text { traveling }\end{array}$ & $\begin{array}{l}\text { distance or traveling time plus a } \\
\text { bonus when the agent is tired }\end{array}$ \\
\hline
\end{tabular}

This generation of the described feeling is done to avoid such bad situations. It is necessary to store them as feelings to have an appropriate solution at hand when a decision must be taken. Without that the calculation of recovering the last good and bad situations with the worst and the best situation evaluation might take too long, and we miss the next tram. Missing the tram is also a situation that must be avoided that 
we learned when missing an important event in that context.

However, feelings are not only pre-calculated evaluations of evaluations. They are also representing our current state and set it in relation to our experiences. So when we are traveling by car, and we are late, we relate the feeling of missing the important event with aggression. This feeling activates an aggressive way of driving and leads to general aggressive behavior in that situation.

In opposite to just using the distance or the estimated time of travel as the weight of the edges in the data model for navigation, we would like to suggest using scores for each edge. In Fig. 4 a fictive road network and three possible variants of route selection are depicted. In Fig. 4a there are five different scores mentioned. Each score is a formula learned over time by experiencing different situations, incorporating multiple inputs. In Table 1 examples of the different scores are listed.

Depending on the emotions from the primary process (happiness, anger, anxiety, sadness, elation and satiation) [13] the formula for each score might result in a different value. There are aggressive and libidinous emotions. For example, if there are lots of aggressive emotions, the agent will decide to use the motorway instead of a romantic street with lots of serpentines and bridges with overlooks. When the agent is in a hurry, the Motorway and the segment with the tunnel might have a benefit. When the agent is tired, the Car slepper route has a benefit, and the segment with the tunnel might have a drawback. In Table 2 the dependency between primary process emotion situation and selected route from Fig. 4 is outlined.

Table 2. Dependency Between Primary Process Emotion Situation and Selected Route

\begin{tabular}{|l|l|}
\hline Primary process emotion situation & Selected Route \\
\hline Agent is not aggressive and in hurry & $\begin{array}{l}\text { Route No. 1: normal street and long tunnel with } \\
\text { two-way traffic. Depicted in Fig. 4b }\end{array}$ \\
\hline Agent is not aggressive, in relaxing mood and tired & $\begin{array}{l}\text { Route No. 2: normal street, serpentines and bridges } \\
\text { and the car slepper train. Depicted in Fig. 4c }\end{array}$ \\
\hline $\begin{array}{l}\text { Agent is aggressive, maybe a little bit in hurry and } \\
\text { tired }\end{array}$ & $\begin{array}{l}\text { Route No. 3: Motorway and Car slepper train } \\
\text { Depicted in Fig. 4d }\end{array}$ \\
\hline
\end{tabular}

\section{Conclusion and Outlook}

We have shown a way to make the localization and navigation model in the project SiMA showing more human-like behavior. Initially, there was the opinion that the navigation is a purely secondary process located procedure, which turned out being wrong. Especially the shown mechanic of incorporating the mood of the agent into the route selection is a very reasonable way of human navigation. This method of navigation will in the next step get integrated into the multi-agent simulation framework of the project SiMA, which is continuously improved and developed further, e.g. in [14], to fulfill further tasks.

\section{References}

[1] Dietrich, D. (2000). Evolution potentials for Feldbus systems, factory communication systems. Proceedings of IEEE International Workshop on Bd: Vol 1 (pp. 145-146).

[2] Bionics. (2016). Retrieved from http://www.britannica.com/technology/bionics

[3] Brandstaetter, C., Schaat, S., Wendt, A., \& Fittner, M. (2017). How agents use breadcrumbs to find their Way. Journal of Computers, 12(1), 89-96.

[4] Fromm, E., \& Funk, R. (2013). The revision of psychoanalysis. Open Road Media.

[5] Akhtar, S. (2009). Comprehensive dictionary of psychoanalysis. Karnac Books.

[6] Freud, S. (1900). The standard edition of the complete psychological works of sigmund freud. The Interpretation of Dreams. 
[7] Langs, R. (2009). Freud on a precipice: How Freud's fate pushed psychoanalysis over the edge. Jason Aronson.

[8] Freud, S. (1923). The Ego and the Id. The Standard Edition of the Complete Psychological Works of Sigmund Freud, 1-66.

[9] Schaat, S. (2016). Simulation of Foundational Human Information-Processing in Social Context.

[10] Lang, R. (2010). A Decision Unit for Autonomous Agents Based on the Theory of Psychoanalysis.

[11] Tolman, E. (1948). Cognitive maps in rats and men. Psychological review, 55(4), 18.

[12] Dietrich, D., Jakubec, M., Schaat, S., Doblhammer, K., Fodor, G., \& Brandstätter, C. (2017). The fourth outrage of man - is the turing-test still up to date? Journal of Computers, 12(2), 116-126.

[13] Schaat, S., Wendt, A., Jakubec, M., Gelbard, F., Herret, L., \& Dietrich, D. (2014). ARS - An AGI Agent Architecture. Proceedings of the 7th International Conference on Artificial General Intelligence (pp. 155-164). Cham: Springer International Publishing.

[14] Wendt, A. (2016). Experience-Based Decision-Making in a Cognitive Architecture.

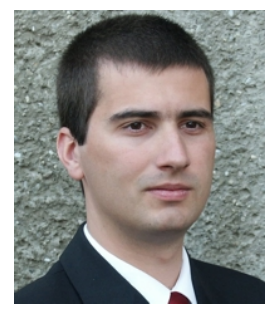

Christian Brandstätter was born in 1979 at Tulln, Austria, studied informatics and informatics-management at the Vienna University of Technology and finished both studies with the degree master of science.

He works as system administrator for general IT in a pharmaceutical research institute. His responsibility there is the IT infrastructure (network, storage, and security). Currently, he works on his dissertation and is a member of the Institute of Computer Technology at the Vienna University of Technology. Brandstätter is a member of IEEE.

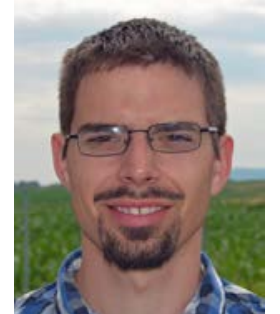

Martin Fittner was born in 1984 at Mistelbach, Austria, studied embedded systems at the University of Applied Science Technikum Wien in Vienna and finished with the degree master of science.

He works as a senior developer for software and system design for automotive electronic systems at the Robert Bosch AG. He is a member of the SiMA (Simulation of the Mental Apparatus and Applications) team. Currently, he works on his dissertation at the Vienna University of Technology in the field of the SiMA project. 